\title{
Memories to Stanisław Kielich: My teacher, my mentor and my friend
}

\author{
Tadeusz Bancewicz* \\ Nonlinear Optics Division, Faculty of Physics, Adam Mickiewicz University, Umultowska 85, 61-614 \\ Poznań, Poland
}

\section{Kielich's style}

There is no doubt that Kielich wrote his papers in his own elegant and specific style. He invented and introduced a special formalism suitable for molecular and nonlinear physics based on Cartesian tensors in which he made use of his deep knowledge of achievements of the old masters of molecular physics. In his scientific work he has shown his great involvement in molecular physics. In the peak of his carrier he wrote his papers fast and efficiently. In the nineteen sixties after construction of the laser, a lot of new optical (linear and nonlinear) effects were discovered. Several of them Kielich had already on his mind. His colleges remember that at a few occasions on the day following their discussions of a new physical effect Kielich would come to the Institute with his paper on the subject almost ready for publication. He mastered tensor calculus, so nonlinear optics was the area he admired. He learned tensor calculus while working with Piekara [1] and then he extended this formalism. From the early years of his scientific carrier he strongly admired works of Buckingham $[2,3]$. He liked to generalize every problem he worked on.

\section{Light scattering}

Kielich was especially interested in the theory of light scattering (linear and nonlinear). One of his first single-handed papers was devoted to the light scattering problems [4-7]. Moreover, from the beginning of his research work he was strongly involved in several subjects of nonlinear optics [8]. It is interesting to note that the laser had yet not been constructed at this time! Invention of the laser was a great stimulation in the area of nonlinear physics research. Already in 1961 Kielich wrote a paper on the nonlinear light scattering [9]. Several works followed this study [10-17]. Kielich is recognized [10,12] for having predicted for the first time the phenomenon of the hyper-Rayleigh scattering of intense laser

\footnotetext{
${ }^{*}$ Corresponding author. E-mail: tbancewi@zon12.physd.amu.edu.pl.
} 


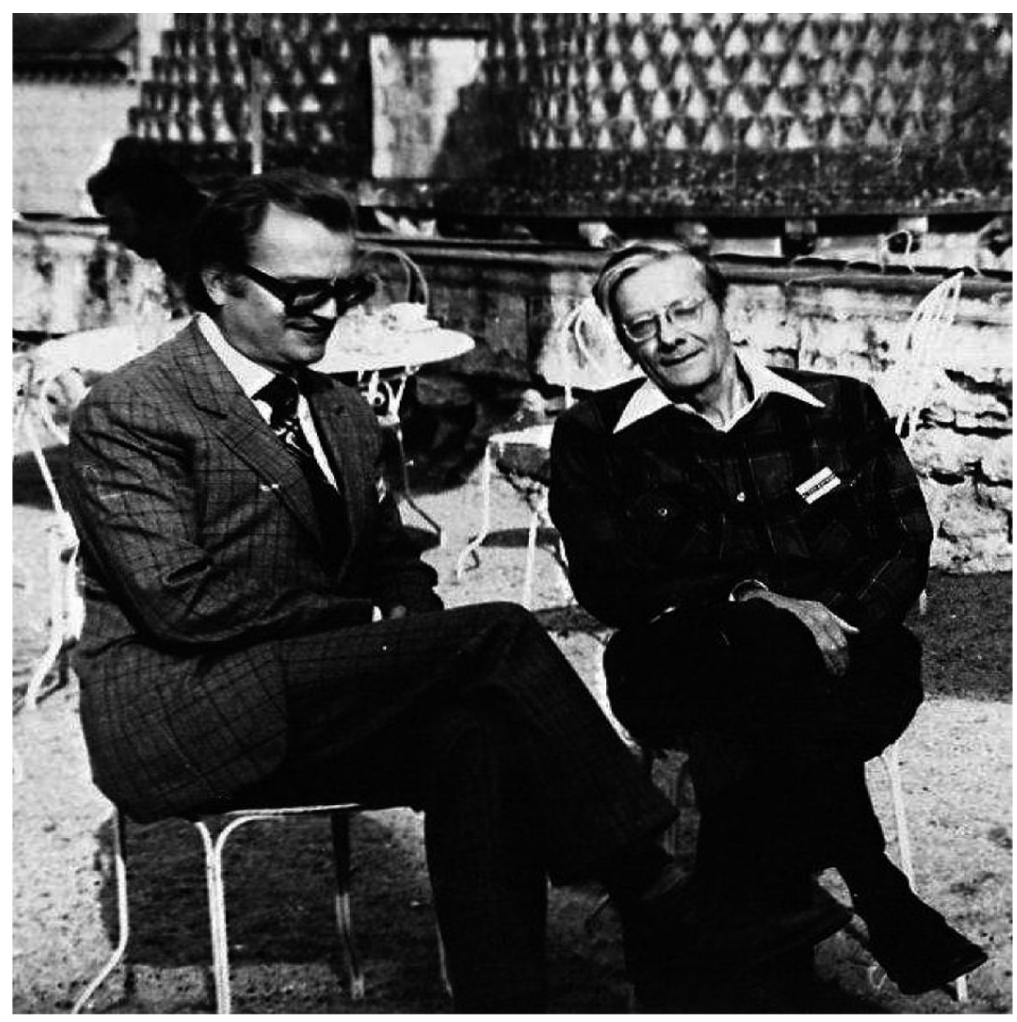

Fig. 1. Professor Kielich with Professor Bloembergen in Fontevrault.

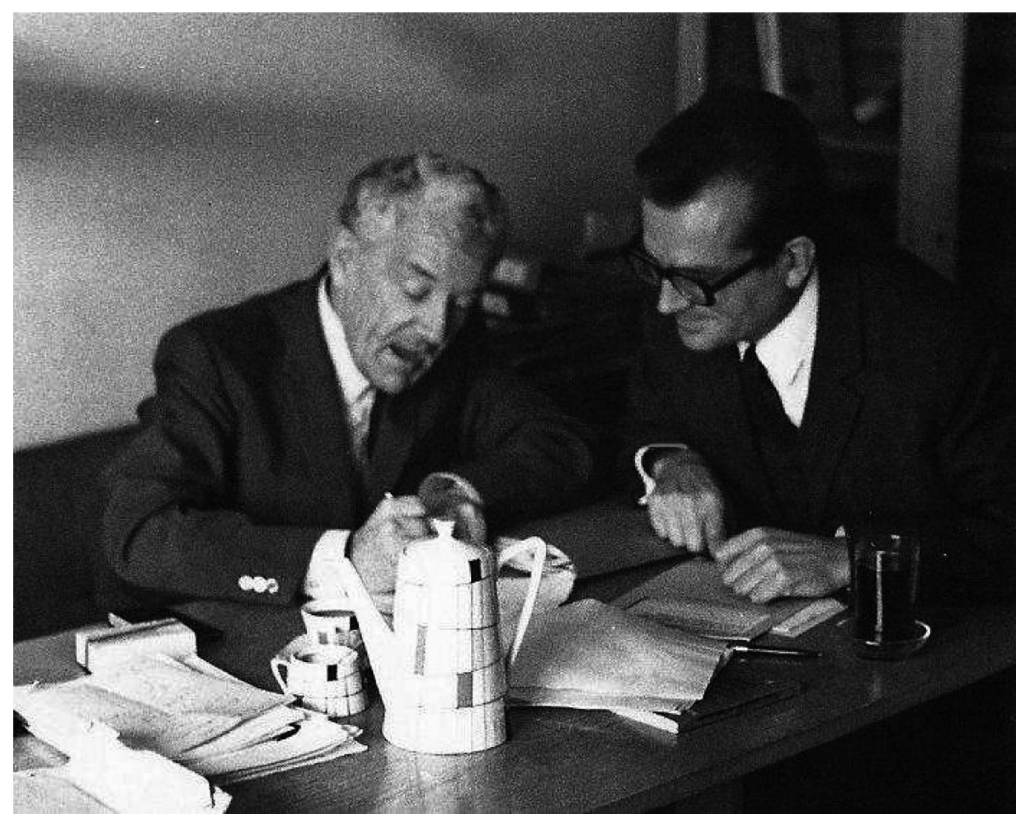

Fig. 2. Professor Kielich with Professor Davies working in Poznañ. 
light. Parallel studies were performed by Li [18] and Cyvin, Rauch and Decius [19]. This effect was experimentally detected for the first time in 1965 by Terhune, Maker and Savage [20].

Studying light scattering process Kielich paid special attention to the role of intermolecular interactions in nonlinear phenomena [17,21-23]. He studied intermolecular interactions in his very elegant and efficient statistical-molecular tensor based style [24]. In 1968 the monomer forbidden Rayleigh collisioninduced light scattering was detected [25,26]. It was shown that an interacting pair of spherical molecules (atoms) possesses an excess anisotropic collision-induced polarizability and this excess polarizability leads to monomer forbidden depolarized light scattering. Kielich was strongly convinced that similar process exists also in the case of the hyper-Rayleigh (and hyper-Raman) light scattering. He shown that colliding pair of (even) centrosymmetric molecules has configurations with no center of inversion and the created fly-by supermolecule is a source of the second harmonic light scattering. This kind of scattering was detected by Kielich, Lalanne and Martin [27] during Kielich's sabbatical (1971-1972) year at the Bordeaux University.

\section{Other subjects}

Kielich has also been involved in many other scientific subjects. The number of subjects he studied is impressive. He started his scientific carrier studying statistical molecular theories of electric, magnetic, and optical saturation (with A. Piekara). Then he investigated intermolecular multipolar interactions in electro-optical and magneto-optical phenomena including optical activity. He considered linear and nonlinear spectroscopy of macromolecular and colloidal systems. He introduced to this field special functions now named Langevin-Kielich functions. Kielich contributed also to quantum optics. He studied photon anti-bunching and squeezed states of the electromagnetic field. All Kielich's papers are available in the pdf format on the web page http://zon8.physd.amu.edu.pl in the section: history.

Institutions and laboratories have a history which influence their future. Some scientists contribute strongly to this history. Stanisław Kielich was one of those.

\section{References}

[1] A. Piekara and S. Kielich, A non-linear theory of the electric permittivity and refractivity of dielectric liquids in electric and magnetic fields, Acta Phys Polon 17 (1958), 209-238.

[2] A.D. Buckingham, Permanent and induced molecular moments and long-range intermolecular forces, Adv Chem Phys 12 (1967), 107-142.

[3] Optical, Electric and Magnetic Properties of Molecules: A review of the work of A. D. Buckingham, (Elsevier, Amsterdam, 1997) edited by D.C. Clary and B.J. Orr.

[4] S. Kielich, Molecular interaction in the classical theory of light scattering Bull. de L'Academie Polonaise des Sciences Serie des sciences, Math, Astr et Phys 6 (1958), 215-221.

[5] S. Kielich, A molecular theory of light scattering in gases and liquids, Acta Phys Polon 19 (1960), 149-178.

[6] S. Kielich, Molecular theory of light scattering by multi-component systems, Acta Phys Polon 19 (1960), 573-597.

[7] S. Kielich, Rayleigh's ratio and turbidity of imperfect gases, Acta Phys Polon 19 (1960), 711-730.

[8] S. Kielich, Semi-macroscopic treatment of the theory of non-linear phenomena in dielectric liquids submitted to strong electric and magnetic fields, Acta Phys Polon 17 (1958), 239-255.

[9] S. Kielich, Theory of light scattering by nonlinear deformable spherical molecules of a gas, Bull Soc Amis Sci Lettres (Poznan) 16 (1961), 69-80.

[10] S. Kielich, On non-linear light scattering in gases, Acta Phys Polon 23 (1963), 321-332.

[11] S. Kielich, Classical theory of nonlinear molecular light scattering, Bull Soc Amis Sci Lettres (Poznan) 17 (1962-1963), 43-58.

[12] S. Kielich, Theory of molecular light scattering in the presence of an intense light beam, Acta Phys Polon 24 (1964), $135-154$. 
[13] S. Kielich, Influence of a strong optical electric field on the molecular scattering of light, Bull Soc Amis Sci Lettres (Poznan) 30 (1964), 1717-1724.

[14] S. Kielich, Sur la diffusion de la lumiere en presence d'un champ intense, electrique ou magnetique, Acta Phys Polon 23 (1965), 819-841.

[15] S. Kielich, Nonlinear light scattering by molecules without a centre of inversion Bull. de L'Academie Polonaise des Sciences Serie des sciences, Math, Astr et Phys 23 (1964), 53-60.

[16] S. Kielich, Non-linear processes to result from multipole interaction between molecules and electromagnetic fields, Proc Phys Soc 86 (1965), 709-725.

[17] S. Kielich, Role of molecular interaction in anisotropic light scattering by liquids, J Chemical Physics 46 (1967), 4090-4099.

[18] Y.Y. Li, Acta Physica Sinica 20 (1964), 164.

[19] S.J. Cyvin, J.E. Rauch and J.C. Decius, Theory of Hyper-Raman Effects (Nonlinear Inelastic Light Scattering): Selection Rules and Depolarization Ratios for the Second-Order Polarizability, J Chem Phys 43 (1965), 4083-4096.

[20] W. Terhune, P.D. Maker and C.M. Savage, Measurements of Nonlinear Light Scattering, Phys Rev Lett 14 (1965), 681-684.

[21] S.Kielich, The role of molecular multipole interactions in the electric polarization of multi-component systems: I. distortion polarization, Acta Phys Polon 27 (1965), 305-322.

[22] S. Kielich, The role of molecular multipole interactions in the electric polarization of multi-component systems. ii. orientation polarization, Acta Phys Polon 28 (1965), 95-121.

[23] S. Kielich, Second harmonic scattering by dense isotropic media, Acta Phys Polon 33 (1968), 89-104.

[24] S. Kielich, Frequency doubling of laser light in an isotropic medium with electrically destroyed centre of inversion, Opto-Electronics 2 (1970), 5-20.

[25] J.P. McTague and G. Birnbaum, Collision-Induced Light Scattering in Gaseous Ar and Kr, Phys Rev Lett 21 (1968), 661-664.

[26] W. Holzer and Y. Le Duff, Collision-Induced Light Scattering Observed at the Frequency Region of Vibrational Raman Bands, Phys Rev Lett 32 (1974), 205-208.

[27] S. Kielich, J.R. Lalanne and F.B. Martin, Double-photon elastic light scattering by liquids having centrosymnetric molecules, Phys Rev Lett 26 (1971), 1295-1298. 